\title{
Toll-like receptor polymorphisms and cerebral malaria: TLR2 $\triangle 22$ polymorphism is associated with protection from cerebral malaria in a case control study
}

\author{
Jennifer A Greene ${ }^{1}$, Nadia Sam-Agudu², Chandy C John², Robert O Opoka ${ }^{3}$, Peter A Zimmerman ${ }^{1}$ and \\ James W Kazura ${ }^{1 *}$
}

\begin{abstract}
Background: In malaria endemic areas, host genetics influence whether a Plasmodium falciparum-infected child develops uncomplicated or severe malaria. TLR2 has been identified as a receptor for $P$. falciparum-derived glycosylphosphatidylinositol (GPI), and polymorphisms within the TLR2 gene may affect disease pathogenesis. There are two common polymorphisms in the $5^{\prime}$ un-translated region (UTR) of TLR2, a 22 base pair deletion in the first unstranslated exon ( $\Delta 22)$, and a GT dinucleotide repeat in the second intron (GTn).

Methods: These polymorphisms were examined in a Ugandan case control study on children with either cerebral malaria or uncomplicated malaria. Serum cytokine levels were analysed by ELISA, according to genotype and disease status. In vitro TLR2 expression was measured according to genotype.

Results: Both $\Delta 22$ and GTn polymorphisms were highly frequent, but only $\Delta 22$ heterozygosity was associated with protection from cerebral malaria (OR $0.34,95 \%$ confidence intervals $0.16,0.73$ ). In vitro, heterozygosity for $\Delta 22$ was associated with reduced pam3cys inducible TLR2 expression in human monocyte derived macrophages. In uncomplicated malaria patients, $\Delta 22$ homozygosity was associated with elevated serum IL-6 $(p=0.04)$, and long GT repeat alleles were associated with elevated TNF ( $p=0.007)$.
\end{abstract}

Conclusion: Reduced inducible TLR2 expression may lead to attenuated pro-inflammatory responses, a potential mechanism of protection from cerebral malaria present in individuals heterozygous for the TLR2 $\triangle 22$ polymorphism.

Keywords: Malaria, Toll-like receptor, Plasmodium falciparum, Polymorphism, GPI

\section{Background}

In some holoendemic countries, individuals are bitten by malaria-infected mosquitoes 60-300 times a year [1,2]. The most common form of malaria in endemic countries is uncomplicated malaria, characterized by fever, chills, sweats, headaches and nausea. Cerebral malaria is a severe neurologic complication of Plasmodium falciparum infection that occurs much less commonly than uncomplicated malaria. Genetic factors influence

\footnotetext{
*Correspondence: jxk14@case.edu

'Case Western Reserve University, Wolstein Research Building, 2103 Cornell Rd, Cleveland, OH 44106, USA

Full list of author information is available at the end of the article
}

infection outcome, and polymorphisms in genes encoding proteins involved in disease pathogenesis are strong candidates for disease association studies. As a receptor for parasite derived GPI, TLR2 is likely involved in disease pathogenesis [3], however, a previous study examining single nucleotide polymorphisms (SNPs) within TLR2 could not detect an association with severe disease because these SNPs were absent in the populations studied [4]. Reportedly, TLR2 SNPs are absent in some African countries as well, but insertion deletion polymorphisms within the 5' un-translated region of TLR2 are very common [5,6]. A $22 \mathrm{bp}$ insertion/deletion polymorphism (TLR2 $\triangle 22)$ in the first un-translated exon

\section{Biomed Central}


was highly polymorphic in Kenyans and in a Japanese population but was not associated with a disease phenotype $[5,7]$. A GT dinucleotide repeat that varies by approximately 12 to 30 repeats $\left(G_{n}\right)$ is present within the second intron, approximately $100 \mathrm{bp}$ upstream of the translational start site. Repeats of varying length have been associated with susceptibility to tuberculosis, reversal reactions in leprosy and colorectal cancer [8-10]. These polymorphisms are associated with altered in vitro phenotypes. The TLR2 $\Delta 22$ polymorphism was associated with reduced constitutive luciferase reporter activity compared to a construct containing the insertion allele [7]. Shorter $\mathrm{GT}_{\mathrm{n}}$ repeats are associated with reduced TLR2 reporter activity and TLR2 surface expression in vitro [8]. Altered TLR2 expression in individuals with different TLR2 polymorphisms may lead to differential pro-inflammatory responses based on genotype. TLR2 expression on human monocytes ex vivo is highly variable [11]. Reports on pam3cys and LPS inducible TLR2 expression on human monocytes vary, likely due to donor specific differences as monocytes differentiate into macrophages [11,12]. Plasmodium falciparum derived GPI activate TLR2 to induce TNF, IL-12, IL- 6 and nitric oxide in murine bone marrow derived macrophages [3]. Elevated levels of IFN- $\gamma$, TNF, IL-12, IL-6, IL-1 $\beta$, and IL-10 are initially protective, although excessive serum levels of these cytokines are associated with cerebral malaria pathology [13-15]. Similarly, IFN- $\gamma$ and TNF are necessary for disease in the murine model of cerebral malaria [16-19]. In the children examined in this study, cerebral malaria patients had elevated serum levels of IFN- $\gamma$, IL-1 $\beta$, and IL-10 compared to uncomplicated malaria patients [20]. Although neither IL-6 nor TNF serum levels were correlated with severe disease in these children [20] these cytokines are often associated with severe disease in other epidemiologic studies [13].

In the current study, the role of TLR2 insertion/deletion polymorphisms, $\Delta 22$ and $\mathrm{GT}_{\mathrm{n}}$, in the pathogenesis of cerebral malaria was evaluated. Genomic DNA and serum were collected from Ugandan children participating in a case control study examining children with cerebral malaria and uncomplicated malaria. This data indicates heterozygosity for $\Delta 22$ polymorphism was protective (odds ratio $0.34,95 \%$ CI $0.16-0.73$ ), but there was no disease association with the $\mathrm{GT}_{\mathrm{n}}$ polymorphism. In malaria naïve donors, $\Delta 22$ heterozygosity was associated with reduced pam3cys induced TLR2 stimulation in human monocyte derived macrophages. In Ugandan children involved in the case control study, none of these polymorphisms was associated with serum cytokines in the cerebral malaria group. In the uncomplicated malaria group, homozygosity for the $\Delta 22$ insertion allele was associated with elevated IL-6 $(p=0.04)$, and at least one long GT repeat allele was associated with elevated serum TNF levels $(p=0.007)$. This data suggests that the TLR2 insertion deletion polymorphisms may be involved in cerebral malaria pathogenesis through alteration of induced TLR2 expression and down-regulation of specific pro-inflammatory cytokines.

\section{Methods}

Children aged three-12 years were recruited for a study assessing complications of cerebral malaria, which was conducted at Mulago Hospital in Kampala, Uganda [20]. Eighty-five children with cerebral malaria (CM) and 76 children with uncomplicated malaria (UM) were recruited. Children with $\mathrm{CM}$ were included in the study if they were admitted to Mulago Hospital, and met the WHO criteria for CM: coma (Blantyre coma scale $\leq 2$, Glasgow coma scale $\leq 8), P$. falciparum-smear positive, and no other signs of encephalopathy. Lumbar puncture was performed to rule out meningitis or encephalitis. Children with UM were enrolled at the acute-care clinic or a malaria outpatient clinic that is sponsored by the University of California San Francisco (UCSF). Children were enrolled as UM patients if they exhibited the signs and symptoms of malaria (fever, chills, vomiting, headache), were $P$. falciparum-smear positive, had no malaria-related complications (e.g. seizures, coma, severe anaemia, or respiratory distress), no other sign of acute illness, and were not hospitalized for their malarial illness. Details of study enrolment have been reported previously [20]. Genetic testing was requested from study participants upon follow-up testing at two years after enrolment. If consent was obtained from the study participant parent or guardian, testing for TLR polymorphisms was performed on the filter paper samples collected at initial enrolment.

Exclusion criteria for children in all three groups was (1) a history of meningitis or encephalitis, or any other brain disorder (including CM); (2) a history of developmental delay; (3) prior admission to the hospital for malnutrition; (4) a history of chronic illness. Ethical approval for these studies was granted by the institutional review boards for human studies at Makerere University Faculty of Medicine, University Hospitals of Cleveland, Case Western Reserve University, Indiana Wesleyan University, and the University of Minnesota. Upon admission, study participants' blood was collected onto Whatman filter paper (Whatman Corporation, Florham Park, NJ). Genomic DNA was extracted from dried blood spots using the QIAamp 96 spin blood kit (QIAGEN, Valencia, CA). Presence of P. falciparum was determined by light microscopy of thin and thick blood smear with two independent readings. A third independent reading was performed if necessary to resolve any discrepancies between the initial two readings. 


\section{Polymerase chain reaction (PCR)}

Genomic DNA was extracted from blood using the QIAamp 96 spin blood kit (QIAGEN, Valencia, CA). PCR was performed using a master mix consisting of $1 \times$ PCR buffer, $125 \mu \mathrm{M}$ dNTPs, $2.5 \mathrm{mM} \mathrm{MgCl}_{2}, 125 \mathrm{nM}$ primers, and 0.8 units Taq polymerase in a reaction volume of $25 \mu \mathrm{l}$. PCR primers and amplification conditions have been described previously [5]. TLR2 $\Delta 22$ genotypes were assigned based on size discrimination of PCR products on a $4 \%$ agarose gel. TLR2 $\mathrm{GT}_{\mathrm{n}}$ genotypes were assigned based on size discrimination of PCR products run on a $6 \%$ polyacrylamide gel as described elsewhere [21].

\section{Cloning and sequencing}

PCR amplification products from local donors were purified using the QIAquick PCR purification kit (QIAGEN, Valencia, CA). Purified PCR products were sent to MWG Biotech, High Point, NC for sequencing. Sequences were analysed using the Sequencher software (Gene Codes Corporation, Ann Arbor, MI).

\section{Cytokine testing}

Serum levels of IFN $\gamma$, TNF, IL-1 $\beta$, IL-10, and IL6 in children with uncomplicated malaria and cerebral malaria were compared according to TLR2 genotype. Serum samples for cytokine measurement were obtained at the time of admission (CM) or outpatient enrollment (UM). Cytokine testing was done using cytometric bead array (CBA) technology using the Bioplex-Luminex system (Austin TX), or ELISA as previously described $[6,20]$.

\section{Statistical analysis}

Allele and genotype frequencies were calculated for the different polymorphisms, and the Hardy-Weinberg exact test (estimation of p-values by the Markov chain method) was performed for each population using Arlequin version 3.01[22]. Haplotypes were statistically inferred using the Expectation - Maximization method in Arlequin. Chi squared test was used to determine allelic, genotypic, and haplotypic associations using SPSS for Windows (version 13.0). Genotypic tests were performed for additive, dominant, recessive, and heterozygote advantage models. Odds ratios were performed for the different genotype models using an online calculator [23]. All cytokines were log transformed to create a normalized distribution, and comparisons for the different genotype models were made using the $T$-test using SPSS for windows (version 13.0). Because of errors due to multiple comparisons, Bonferroni correction assuming two individuals tests was applied. At a false positive rate of $5 \%$, the adjusted significance threshold becomes $p<$ .025 .

\section{TLR2 expression by flow cytometry}

Venous blood was collected from 18 malaria naïve North American volunteers. Peripheral blood mononuclear cells were isolated by Ficoll-Paque separation (GE Healthcare, Piscataway, NJ) and stained with TLR2FITC (clone TL2.1) and CD14-APC (clone 61D3, eBiosciences, San Diego, CA). Cells were read on an LSRII flow cytometer (BD Biosciences, Franklin Lakes, NJ). Monocytes were distinguished by scatter characteristics and CD14 positivity. Median fluorescence intensity of isotype controls were subtracted from each sample to determine relative TLR2 fluorescence. Ethical approval for these studies was granted by the institutional review board for University Hospitals of Cleveland, Case Western Reserve University.

\section{Cell culture}

Peripheral blood mononuclear cells (PBMC) were cultured in RPMI 10\% human serum AB, 2 mM L-glut, 20 $\mathrm{mM}$ HEPES, and pen/strep. PBMC were plated out at 1 $\times 10^{6}$ cells $/ \mathrm{ml}$ and stimulated with Pam3Cys (200 ng/ $\mathrm{ml}$ ) for $24 \mathrm{~h}$. Human monocytes were isolated by immunomagnetic purification using the Miltenyi monocyte isolation kit II (miltneyi) to obtain unlabelled monocytes which were $80-95 \%$ pure. Purified monocytes were cultured for six days in RPMI supplemented with $2 \mathrm{mM} \mathrm{L-}$ glut, $20 \mathrm{mM}$ HEPES, pen/strep, and 10\% autologous serum to allow differentiation into monocyte derived macrophages. Cells were washed three times with PBS, and adherent cells were removed with cell dissociation buffer (vendor) for flow cytometry.

\section{Parasite culture}

Plasmodium falciparum strain 3D7 parasites were cultured and the parasite life cycle was synchronized as described [24]. Trophozoites and schizonts were obtained by magnetic purification. Late stage parasite cultures were washed three times with Macs buffer (PBS, pH 7.2, 0.5\% FBS, and 2 mM EDTA), and resuspended at a concentration of $2 \times 10^{9}$ cells $/ \mathrm{ml}$. Parasite cultures were passed through Miltenyi LD columns (Miltenyi), allowing uninfected RBCs and rings to pass through the column. Miltenyi columns were washed two times $1 \mathrm{ml}$ Macs buffer, and late stage parasites were eluted with $3 \mathrm{ml} \mathrm{Macs}$ buffer. Trophs/schizonts were washed three times and counted. Schizonts were lysed by sonication ( $40 \mathrm{~V}, 10 \mathrm{mins})$. Following sonication, schizonts were brought up to the appropriate concentration in RPMI. Schizonts or intact trophozoites were added to macrophage cultures at a ratio of 10:1.

\section{RT-qPCR}

Approximately $1 \times 10^{6}$ monocytes were isolated for qPCR. Total RNA was extracted using the PureLink 
RNA Mini Kit (Invitrogen). 100 ng of total RNA was reverse transcribed into cDNA using commercial reagents (Invitrogen) and non-specific Oligo-DT primers. Quantitative PCR was carried out using the following gene specific primers; TLR2 - forward 5'ATTGTGCCCATTGCTCTTTC-3', reverse, 5'CTTCCTTGGAGAGGCTGATG-3'; GAPDH - forward 5'AAGATCATCAGCAATGCCTCCTGC-3', reverse 5'ATGGACTGTGGTCATGAGTCCTTC-3'. Samples were analysed on a 7300 Real Time PCR System (Applied Biosystems), and normalized using the comparative threshold method $[25,26]$. HEK cell mRNA was used as a negative control for TLR2 expression.

\section{Results}

TLR2 $\Delta 22$ and $\mathrm{GT}_{\mathrm{n}}$ genetic association with cerebral malaria

A total of 65 children with cerebral malaria and 52 children with uncomplicated malaria were genotyped for $T L R 2$ insertion-deletion polymorphisms. The $\Delta 22$ polymorphism was highly polymorphic in this study, but there was no significant difference in allele frequency between the two groups $(0.27$ in the cerebral malaria group vs 0.35 in the uncomplicated malaria group, $p=$ $0.20)$. The $\mathrm{GT}_{\mathrm{n}}$ polymorphism was highly polymorphic but similarly distributed between the two groups, as demonstrated in Figure 1.

TLR2 $\triangle 22$ and $\mathrm{GT}_{\mathrm{n}}$ genotype frequencies are displayed in Table 1 . The heterozygote advantage genotype model best fits the $\Delta 22$ polymorphism, as heterozygosity was significantly more common in the uncomplicated malaria group compared to the cerebral malaria group (WD vs DD/WW, $p=.005$ OR 0.34, 95\%CI 0.16-0.73), and this was below the adjusted significance threshold of $p<0.025$. Additionally, in the uncomplicated malaria group there was a slight deviation from Hardy Weinberg Equilibrium ( $p=0.065)$, indicating an excess of heterozygotes in this group. Consistent with how the $\mathrm{GT}_{\mathrm{n}}$ repeats have been analysed previously $[8,27]$, the alleles were classified as short $(\mathrm{S}<16)$, medium $(\mathrm{M}, 16-23)$, and long $(\mathrm{L}>23)$ repeats. All individuals possessed at least one medium length repeat allele. Only SM, MM, and ML genotypes for the $\mathrm{GT}_{\mathrm{n}}$ repeat polymorphism were present in the groups studied here, and these genotypes were similarly distributed between cerebral malaria patients and controls with uncomplicated malaria (Table 1). TLR2 haplotype frequencies are presented in Table 2. All six possible TLR2 haplotypes were present, and similarly distributed in each group. There was no linkage disequilibrium between these two

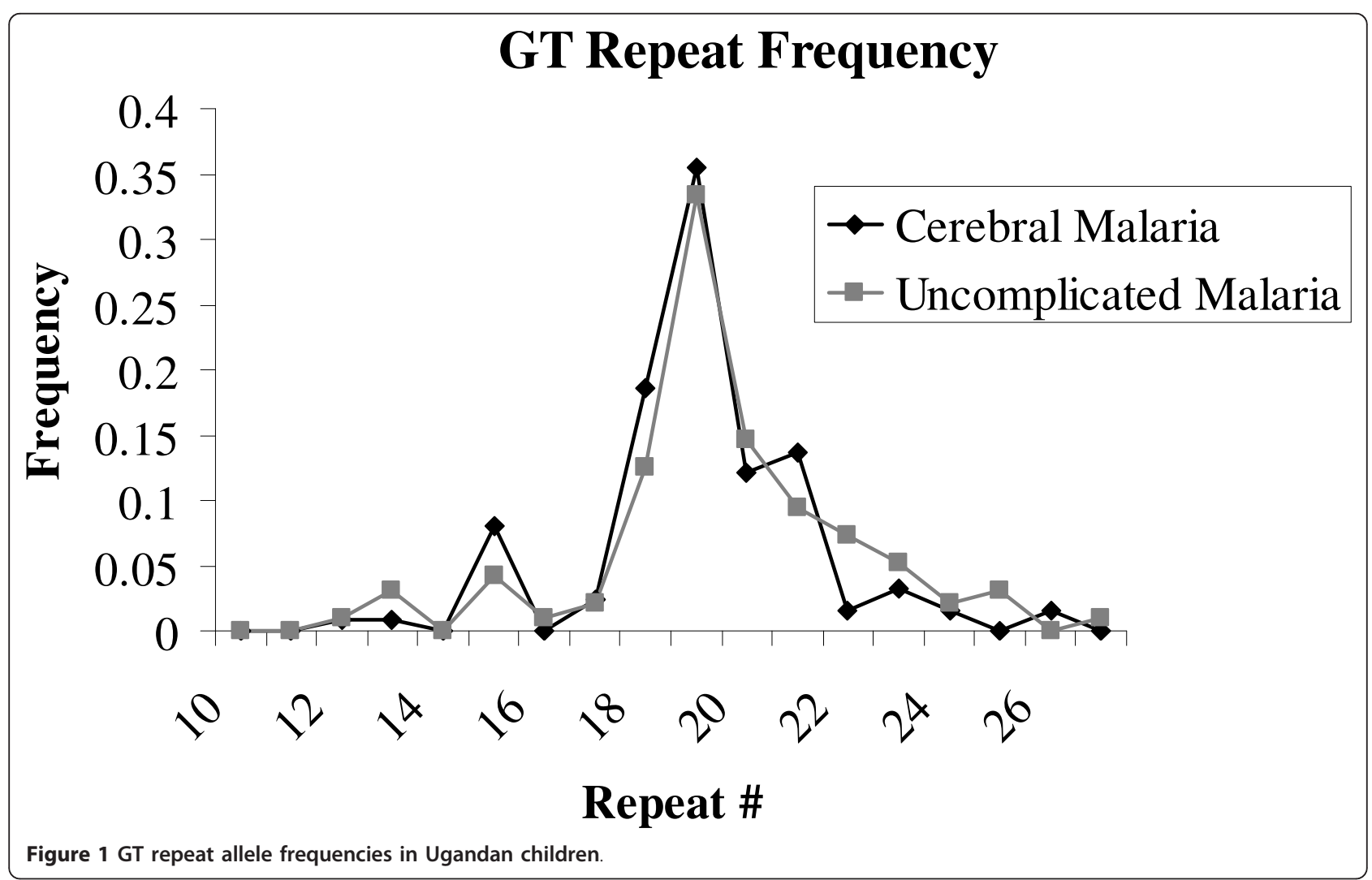


Table 1 TLR2 $\Delta 22$, and $\mathrm{GT}_{\mathrm{n}}$ genotypes

\begin{tabular}{lllllll}
\hline Polymorphism & Genotype & Cerebral malaria $\mathbf{n}=\mathbf{6 5}$ & Uncomplicated malaria $\mathbf{n}=\mathbf{5 2}$ & $\boldsymbol{p}$ value & OR & $\mathbf{9 5 \%}$ Cl \\
\hline$\Delta 22$ & W/W & $36(56.3)$ & $18(35.3)$ & $.025^{1}$ & 0.42 & $0.20-0.98$ \\
& W/D & $21(32.8)$ & $30(58.8)^{\prime \prime}$ & $.005^{\prime \prime \prime}$ & 0.34 & $0.16-0.73$ \\
& D/D & $7(10.9)$ & $3(5.9)$ & & \\
GT & SM & $12(19.4)$ & $8(16.7)$ & & \\
& MM & $46(74.2)$ & $34(70.8)$ & & \\
& ML & $4(6.4)$ & $6(12.5)$ & & \\
\hline
\end{tabular}

OR and $95 \% \mathrm{Cl}$ presented only for $\mathrm{p}$ values where $p<0.05$

${ }^{\prime} \mathrm{p}$ value for dominant genotype test, WD/DD vs WW

" $p=0.065$ for Hardy Weinberg Test of equilibrium, excess of heterozygotes

III $p$ value for heterozygote advantage genotype test, WD vs DD/WW

polymorphisms, as defined by both a Lewontin's D' and $\mathrm{r}^{2}$ value $>0.8\left(\mathrm{UM} \mathrm{D}^{\prime}=0.553, \mathrm{r}^{2}=0.083 ; \mathrm{CM} \mathrm{D}^{\prime}=\right.$ $\left.0.490, r^{2}=0.094\right)$.

\section{Constitutive TLR2 expression on human monocytes and monocyte derived macrophages}

Malaria naïve North American volunteers were recruited and genotyped for TLR2 $\Delta 22$ and $\mathrm{GT}_{\mathrm{n}}$ polymorphisms. TLR2 expression was highly variable on monocytes from local donors ex vivo, and was not associated with $\Delta 22$ genotype in this study (Figure 2). Because of the few donors with small or large GT repeat polymorphisms in this study, the effect of this polymorphism on TLR2 expression on human monocytes ex vivo could not be determined. Reportedly, TLR2 expression on monocytes varies in the first $24 \mathrm{~h}$ of culture, as the cells become adherent and begin differentiating into macrophages $[11,12,28]$. To test whether differentiation dependent TLR2 expression was based on TLR2 $\Delta 22$ genotypes, TLR2 $\mathrm{mRNA}$ and protein expression were determined on monocytes at 0,4 , and $24 \mathrm{~h}$ in culture. Consistent with previous reports, TLR2 expression varied during the first $24 \mathrm{~h}$ in culture, but expression levels were not associated with genotype (Figure 2).

\section{Inducible TLR2 expression}

Because of the variability in TLR2 expression during the first $24 \mathrm{~h}$ of monocyte culture, constitutive and inducible TLR2 expression on human monocyte derived

Table 2 TLR2 haplotypes

\begin{tabular}{lll}
\hline Haplotype & Cerebral malaria & Uncomplicated malaria \\
\hline D S & 0.078 & 0.083 \\
D M & 0.185 & 0.265 \\
D L & 0.000 & 0.006 \\
W S & 0.021 & 0.000 \\
W M & 0.684 & 0.589 \\
W L & 0.033 & 0.057 \\
\hline
\end{tabular}

$\mathrm{D}$, deletion allele, $\mathrm{W}$, wild type

$S<16$ repeats, $M \quad 16-23$ repeats, $L>23$ repeats macrophages was examined. Human monocytes were isolated by negative selection and cultured for six days to allow differentiation into macrophages. Although constitutive TLR2 expression was not associated with $\Delta 22$ genotype (Figure 3), a 24-h Pam3Cys stimulation significantly up-regulated TLR2 expression on macrophages from homozygous donors $(p=0.023)$, but not on macrophages from heterozygous donors $(p=0.20)$. $P$. falciparum strain 3D7 parasites were synchronously cultured to trophozoite stage and added to human monocytes derived macrophages and incubated for $24 \mathrm{~h}$. Neither intact, infected RBCs or uninfected RBCs upregulated TLR2 expression on human monocyte derived macrophages (Figure 3). Lysed infected RBCs were also unable to up-regulate TLR2 expression on human monocyte derived macrophages (data not shown).

\section{Serum cytokine levels in children with different TLR2 polymorphisms}

Serum TNF, IFN- $\gamma$, IL-1 $\beta$, IL-6, and IL-10 did not vary based on either TLR2 $\Delta 22$ or $\mathrm{GT}_{\mathrm{n}}$ genotype in children with cerebral malaria (Figures 4 and 5 and Additional file 1: Table S1). In the uncomplicated malaria group, children with at least one long $\mathrm{GT}_{\mathrm{n}}$ repeat allele produced the most TNF ( $p=0.007$, Figure 5 and Additional file 1: Table S1), and children homozygous for $\Delta 22$ produced the most IL-6 compared to children with other genotypes ( $p=0.041$, Figure 4 and Additional file 1: Table S1). Only five uncomplicated malaria patients had detectable serum IL- $1 \beta$ levels, and they were all heterozygous for $\Delta 22$ and had medium length GT repeat alleles (Figures 4, 5, and Additional file 1: Table S1).

\section{Discussion}

This is the first study to show an association between severe malaria and a TLR2 polymorphism. TLR2 $\Delta 22$ heterozygosity was significantly more common in the uncomplicated malaria group $(p=0.005)$, indicating that the odds of cerebral malaria are $66 \%$ (odds ratio 0.34 , 95\% CI 0.16-0.73) lower in heterozygotes compared to either homozygote. Due to the low frequency of $\Delta 22$ 

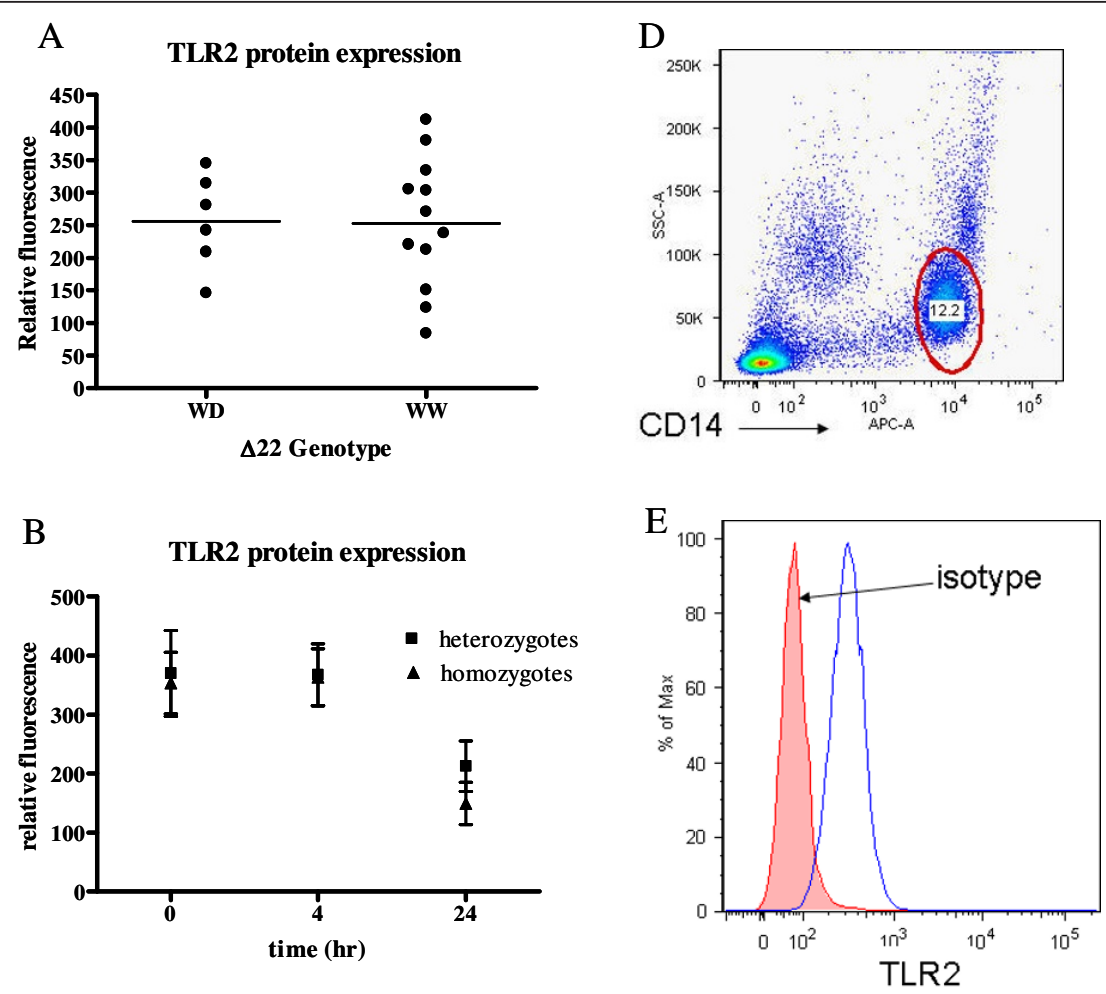

\section{TLR2 mRNA expression}

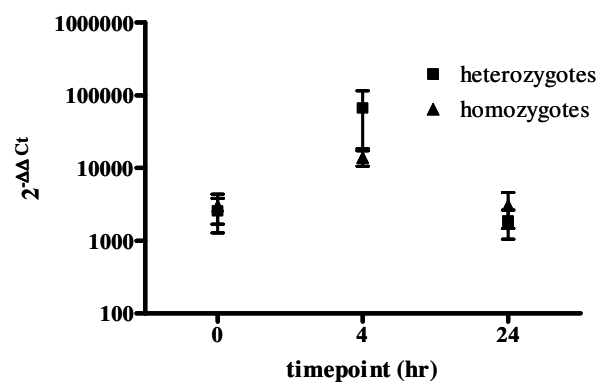

Figure 2 Constitutive TLR2 expression on primary monocytes. TLR2 expression on CD14+ cells immediately ex vivo or during the first $24 \mathrm{~h}$ in culture is shown here. Figure A is TLR2 expression on CD14+ cells ex vivo according to $\triangle 22$ genotype. Relative fluorescence is median fitcTLR2 fluorescence minus median fitc-isotype fluorescence for each individual. Horizontal bars indicate median for each group, and each dot represents one donor. Representative histograms and the gate used to identify monocytes is represented in D and E. Figure B shows TLR2 protein expression on immunopurified monocytes during the first $24 \mathrm{~h}$ in culture according to $\triangle 22$ genotype. Error bars indicate mean $+/-\mathrm{SEM}$. There are four heterozygotes and four homozygotes are in each group. Figure C shows the corresponding TLR2 mRNA expression in the first 24 $\mathrm{h}$ of culture according to TLR2 $\triangle 22$ genotype. Relative mRNA expression was quantified according to the comparative Ct method, using GAPDH as the housekeeping gene, and HEK cell mRNA as a negative control. In graph C the results at $\mathrm{O} \mathrm{h}$ for one heterozygous donor was removed, this person was an outlier, with a $2^{-\Delta \Delta C t}$ value of $3.3 \times 10^{8}$.

homozygotes, and the small sample sizes, the dominant genotype model ( $p=0.025$, OR $0.20-0.90)$ is also a possibility, and future studies with larger samples sizes need to be conducted to more precisely define the role of this polymorphism in susceptibility to cerebral malaria. Future studies should investigate whether this polymorphism is protective against other forms of severe malaria, such as severe malarial anaemia. Individual co- infections may have influenced the frequencies of TLR2 genotypes observed here. The $\Delta 22$ polymorphism was associated with enhanced susceptibility to acute adenolymphangitis events in lymphatic filariasis-infected Papua New Guineans (Greene et al., unpublished results). Lymphatic filariasis is one of many parasitic diseases transmitted in Uganda [29] and the relatively low frequency of $\Delta 22$ homozygotes in the uncomplicated 


\section{TLR2 Protein Expression}

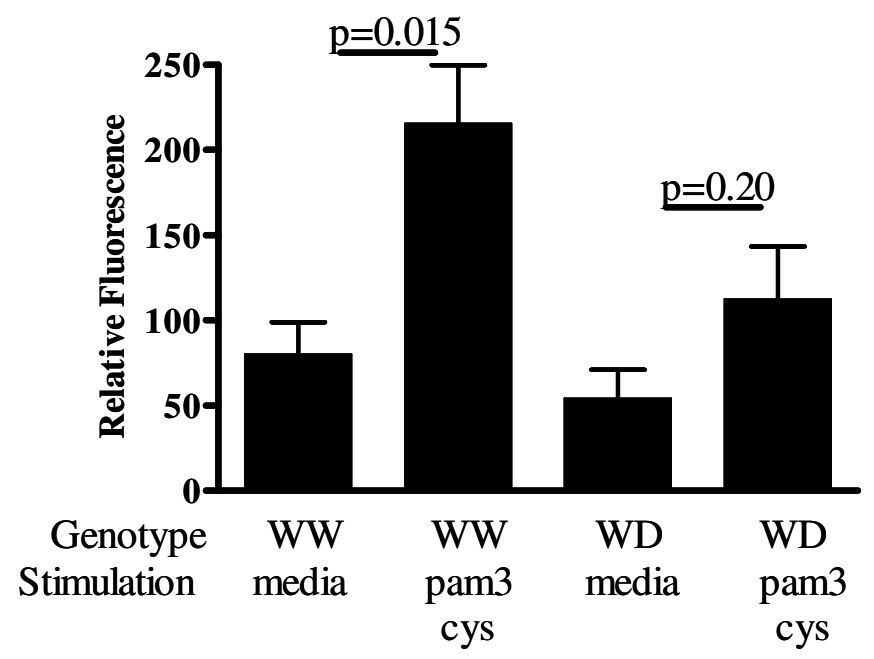

\section{TLR2 protein expression}

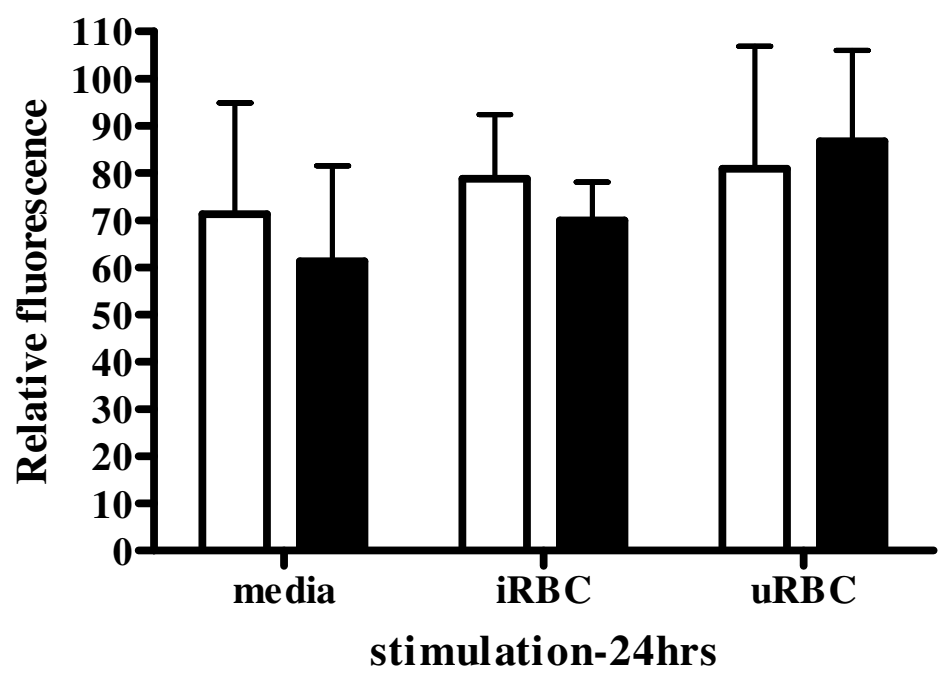

Figure $\mathbf{3}$ Inducible TLR2 expression on macrophages according to TLR2 $\mathbf{\Delta} \mathbf{2 2}$ genotype. Human monocyte derived macrophages were stimulated with pam3cys (200 ng/ml), schizont infected RBCs $\left(3 \times 10^{6} /\right.$ well), or uninfected RBCs $\left(3 \times 10^{6} /\right.$ well) for $24 \mathrm{~h}$. TLR2 protein expression is expressed as relative fluorescence. Top panel $n=5$ homozygotes, $n=5$ heterozygotes, bottom panel, $n=4$ homozygotes, $n=5$ heterozygotes. Error bars represent standard error of the mean.

malaria group may reflect these complex selective pressures in this region.

Within the TLR2 gene, $\triangle 22$ is located in the first untranslated exon of TLR2, approximately 60 bp downstream of an NF-kB site critical for chromatin remodelling and transcription factor binding [28]. The $\mathrm{GT}_{\mathrm{n}}$ repeat is located approximately $100 \mathrm{bp}$ upstream of the transcriptional start site, potentially affecting inclusion of the single coding exon in the transcript. Because of their location within the 5' UTR and proximity to critical cis-elements within the TLR2 gene, it is possible that $\triangle 22$ and $G_{\mathrm{n}}$ may affect TLR2 expression. Although short and long GT repeats were too uncommon in local donors to detect a difference in TLR2 expression, there was reduced TLR2 expression in response to pam3cys in human monocyte derived macrophages. There was a similar, although insignificant hypo-responsiveness in human monocytes from $\Delta 22$ heterozygotes (data not shown). The focus was on macrophages in this study to avoid donor specific effects on monocyte differentiation and subsequent TLR2 expression. There was no significant TLR2 up-regulation in response to either intact 


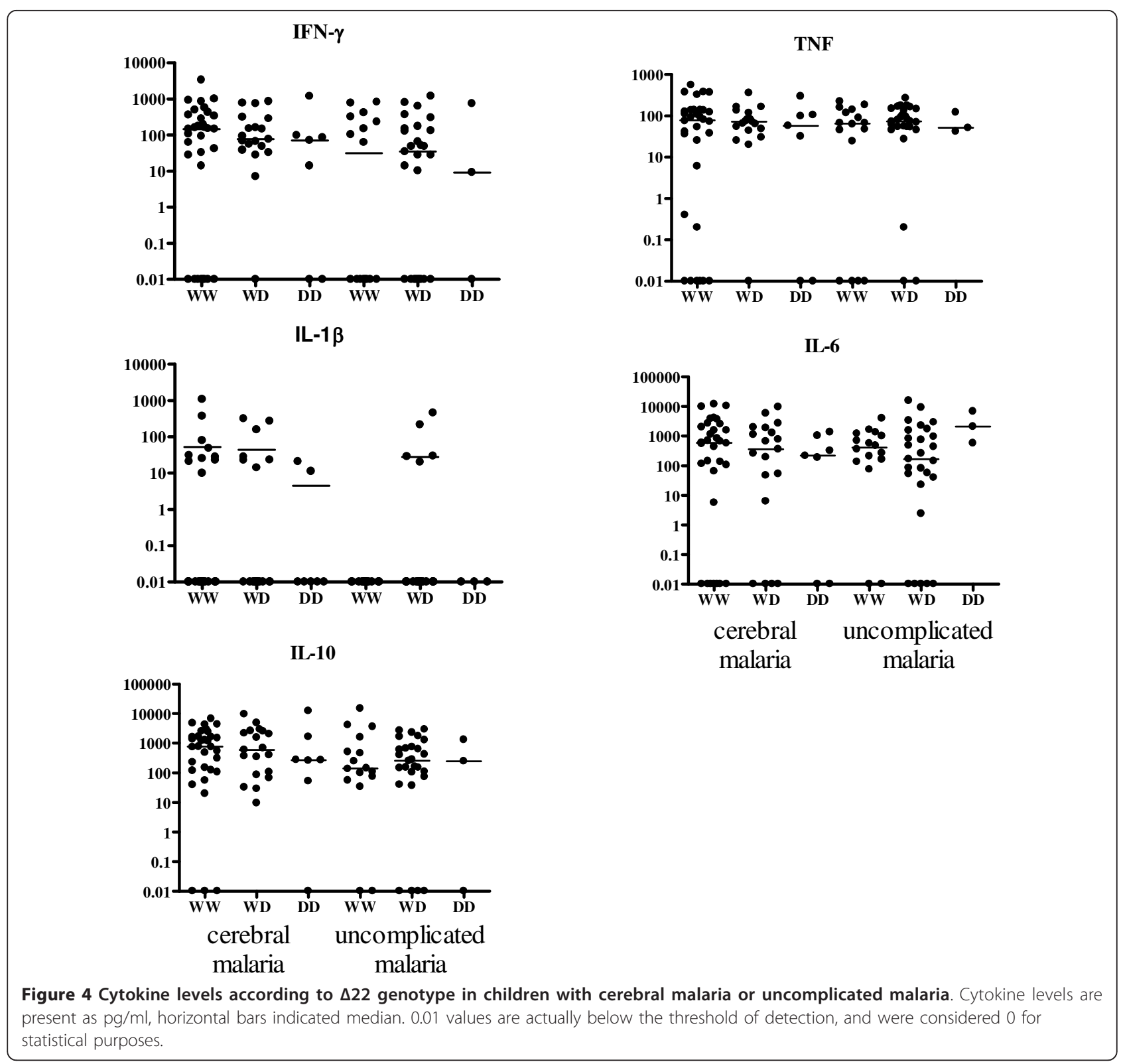

parasitized RBCs or schizont lysate. This does not rule out the possibility that $P$. falciparum may directly upregulate TLR2, but it was not detected in this study, possibly because there was insufficient GPI activating TLR2 in either of the parasite preparations to up-regulate TLR2 expression in a manner similar to pam3cys induction. Future studies should examine whether purified parasite derived GPI up-regulate TLR2. None of the North American donors homozygous for $\Delta 22$, and therefore the effect of this genotype on either constitutive or inducible TLR2 expression could not be determined.

The reduced pam3cys inducible TLR2 expression on macrophages from $\Delta 22$ heterozygous donors suggests that this polymorphism may attenuate TLR2 induced signalling cascades and downstream cytokine production. To test this, serum cytokine responses were examined in the Ugandan children involved in the case control study according to $\Delta 22$ and $\mathrm{GT}_{\mathrm{n}}$ genotypes. There was no significant association between the TLR2 polymorphisms examined here and serum cytokines in the cerebral malaria group. In the uncomplicated malaria group $\Delta 22$ homozygosity was associated with elevated IL-6 production $(p=0.04)$, and at least one long $\mathrm{GT}_{\mathrm{n}}$ allele was associated with elevated serum TNF $(p=0.007)$. In these children, neither IL-6 nor TNF were correlated with severe disease [20], though the relatively small sample size may have precluded 


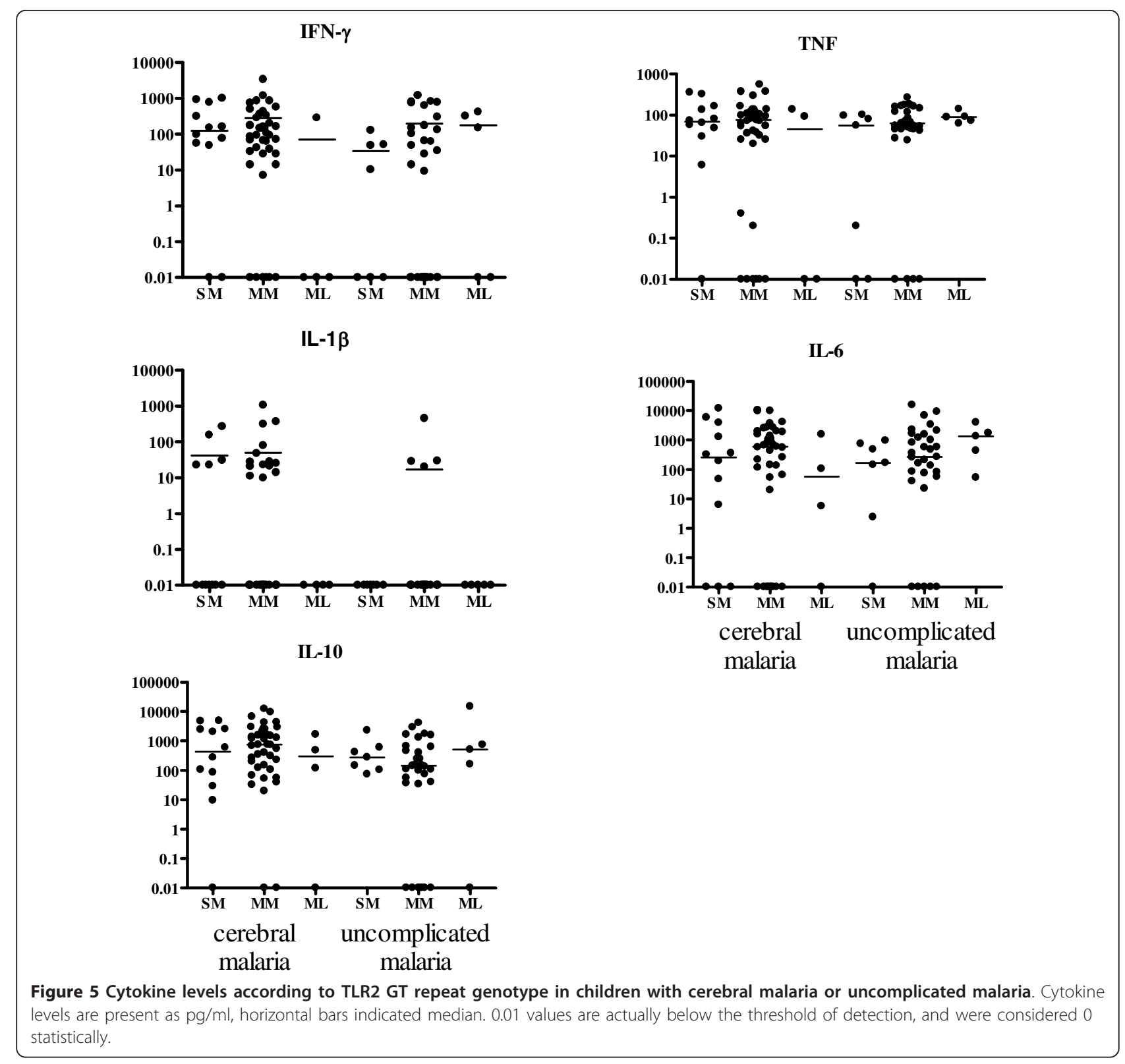

detection of all but large associations. The same limitation regarding sample size applies to the detection of elevated levels of cytokines in children with $\Delta 22$ homozygosity and cerebral malaria. This study should be repeated in an area where elevated IL- 6 and TNF correlate with severe disease. It is possible that cytokine production in individuals with specific TLR2 genotypes may influence development of uncomplicated malaria, and future studies of longitudinal cohorts examining the intensity and frequency of uncomplicated malaria episodes in children with different $\Delta 22$ and $\mathrm{GT}_{\mathrm{n}}$ genotypes may elucidate the relationship between TLR2 genotype and infection outcome. Examining serum cytokines is non-specific, and many cells contribute to serum cytokine levels. Future studies should examine PBMCs from donors with different TLR2 genotypes and measure cytokine responses upon stimulation with intact and lysed infected RBCs. One potentially confounding variable in the current case control study would be the presence of a MAL SNP Ser180Leu. This molecule is a downstream adaptor in the TLR2 signalling pathway, and this polymorphism is associated with protection from malaria and reduced signalling in vitro [30]. The Ugandan samples were genotyped for the MAL polymorphism as described previously [5]. The SNP was present at $1.5 \%$ allele frequency in the cerebral malaria patients, and $1 \%$ allele frequency in the uncomplicated malaria group. Because of the low frequency of this 
SNP, it is unlikely that it had a significant effect on the serum cytokine levels observed here.

\section{Conclusions}

Collectively, this data suggest that heterozygosity for a 22 bp deletion within the first un-translated exon of TLR2 is associated with protection from cerebral malaria, and that this protection may be due to a dampening of the pro-inflammatory response. Pam3Cys inducible TLR2 expression was significantly reduced in macrophages from heterozygous donors. Reduced surface expression may attenuate TLR2 mediated responses. Although there were no significant differences in serum cytokines according to TLR2 genotypes in children with cerebral malaria, future studies examining responses to pam3cys and parasitized RBCs in donors with different TLR2 genotypes will help identify cell responsible for cytokine production.

\section{Additional material}

Additional file 1: Table S1. Cytokine association tests according to TLR2 genotype in Uganda children with cerebral malaria or uncomplicated malaria.

\section{Abbreviations}

RBC: red blood cell; TLR: Toll-Like Receptor; GPI: glycosylphosphatidylinositol; CM: cerebral malaria; UM: uncomplicated malaria; UTR: unstranslated region; OR: odds ratio; SNP: single nucleotide polymorphism.

\section{Acknowledgements}

We gratefully acknowledge Daniel Tisch and Tracy Bergemann for assistance with statistical analysis.

\section{Author details}

${ }^{1}$ Case Western Reserve University, Wolstein Research Building, 2103 Cornell Rd, Cleveland, $\mathrm{OH} 44106$, USA. ${ }^{2}$ University of Minnesota, Minneapolis, MN, USA. ${ }^{3}$ Makerere University, Kampala, Uganda.

\section{Authors' contributions}

JG performed genotyping, in vitro work, statistical analysis, and drafted manuscript. JXK and CCJ conceived and designed the study. PAZ was involved in data analysis. NSA conducted ELISAs. ROO was involved in study sample collection. All authors read and approved the final manuscript.

\section{Competing interests}

The authors declare that they have no competing interests.

Received: 7 October 2011 Accepted: 15 February 2012

Published: 15 February 2012

\section{References}

1. CDC:[http://www.cdc.gov/malaria/facts.htm].

2. Newton $C R$, Hien TT, White N: Cerebral malaria. J Neurol Neurosurg Psychiatry 2000, 69:433-441.

3. Krishnegowda G, Hajjar AM, Zhu J, Douglass EJ, Uematsu S, Akira S, Woods AS, Gowda DC: Induction of proinflammatory responses in macrophages by the glycosylphosphatidylinositols of Plasmodium falciparu: cell signaling receptors, glycosylphosphatidylinositol (GPI) structural requirement, and regulation of GPI activity. J Biol Chem 2005, 280:8606-8616.
4. Mockenhaupt FP, Cramer JP, Hamann L, Stegemann MS, Eckert J, Oh NR, Otchwemah RN, Dietz E, Ehrhardt S, Schröder NW, Bienzle U, Schumann RR: Toll-like receptor (TLR) polymorphisms in African children: common TLR4 variants predispose to severe malaria. J Commun Dis 2006, 38:230-245.

5. Greene JA, Moormann AM, Vulule J, Bockarie MJ, Zimmerman PA, Kazura JW: Toll-like receptor polymorphisms in malaria-endemic populations. Malar J 2009, 8:50.

6. Sam-Agudu NA, Greene JA, Opoka RO, Kazura JW, Boivin MJ, Zimmerman PA, Riedesel MA, Bergemann TL, Schimmenti LA, John CC: TLR9 polymorphisms are associated with altered IFN-gamma levels in children with cerebral malaria. Am J Trop Med Hyg 2010, 82:548-555.

7. Noguchi E, Nishimura F, Fukai H, Kim J, Ichikawa K, Shibasaki M, Arinami T: An association study of asthma and total serum immunoglobin $E$ levels for Toll-like receptor polymorphisms in a Japanese population. Clin Exp Allergy 2004, 34:177-183.

8. Yim JJ, Lee HW, Lee HS, Kim YW, Han SK, Shim YS, Holland SM: The association between microsatellite polymorphisms in intron II of the human Toll-like receptor 2 gene and tuberculosis among Koreans. Genes Immun 2006, 7:150-155.

9. Boraska Jelavic T, Barisic M, Drmic Hofman I, Boraska V, Vrdoljak E, Peruzovic M, Hozo I, Puljiz Z, Terzic J: Microsatelite GT polymorphism in the toll-like receptor 2 is associated with colorectal cancer. Clin Genet 2006, 70:156-160.

10. Bochud PY, Hawn TR, Siddiqui MR, Saunderson P, Britton S, Abraham I, Argaw AT, Janer M, Zhao LP, Kaplan G, Aderem A: Toll-like receptor 2 (TLR2) polymorphisms are associated with reversal reaction in leprosy. J Infect Dis 2008, 197:253-261.

11. Haehnel V, Schwarzfischer $L$, Fenton MJ, Rehli M: Transcriptional regulation of the human toll-like receptor 2 gene in monocytes and macrophages. $\mathrm{J}$ Immunol 2002, 168:5629-5637.

12. Henning LN, Azad AK, Parsa KV, Crowther JE, Tridandapani S, Schlesinger LS: Pulmonary surfactant protein A regulates TLR expression and activity in human macrophages. J Immunol 2008, 180:7847-7858.

13. Haldar K, Murphy SC, Milner DA, Taylor TE: Malaria: mechanisms of erythrocytic infection and pathological correlates of severe disease. Annu Rev Pathol 2007, 2:217-249.

14. de Kossodo S, Grau GE: Profiles of cytokine production in relation with susceptibility to cerebral malaria. J Immunol 1993, 151:4811-4820.

15. Kwiatkowski D, Hill AV, Sambou I, Twumasi P, Castracane J, Manogue KR, Cerami A, Brewster DR, Greenwood BM: TNF concentration in fatal cerebral, non-fatal cerebral, and uncomplicated Plasmodium falciparu malaria. Lancet 1990, 336:1201-1204.

16. Amani V, Vigario AM, Belnoue E, Marussig M, Fonseca L, Mazier D, Renia L: Involvement of IFN-gamma receptor-medicated signaling in pathology and anti-malarial immunity induced by Plasmodium berghe infection. Eur J Immunol 2000, 30:1646-1655.

17. Curfs JH, van der Meide PH, Billiau A, Meuwissen JH, Eling WM: Plasmodium berghe: recombinant interferon-gamma and the development of parasitemia and cerebral lesions in malaria-infected mice. Exp Parasitol 1993, 77:212-223.

18. Grau GE, Fajardo LF, Piguet PF, Allet B, Lambert PH, Vassalli P: Tumor necrosis factor (cachectin) as an essential mediator in murine cerebral malaria. Science 1987, 237:1210-1212.

19. Grau GE, Heremans H, Piguet PF, Pointaire P, Lambert PH, Billiau A, Vassalli P: Monoclonal antibody against interferon gamma can prevent experimental cerebral malaria and its associated overproduction of tumor necrosis factor. Proc Natl Acad Sci USA 1989, 86:5572-5574.

20. John CC, Opika-Opoka R, Byarugaba J, Idro R, Boivin MJ: Low levels of RANTES are associated with mortality in children with cerebral malaria. J Infect Dis 2006, 194:837-845.

21. DaRe JT, Mehlotra RK, Michon P, Mueller I, Reeder J, Sharma YD, Stoneking M, Zimmerman PA: Microsatellite polymorphism within pfcrt provides evidence of continuing evolution of chloroquine-resistant alleles in Papua New Guinea. Malar J 2007, 6:34.

22. Arlequin version 3.01. [http://cmpg.unibe.ch/software/arlequin3].

23. Bland JM, Altman DG: Statistics notes The odds ratio. BMJ 2000, 320:1468.

24. Grimberg BT, Erickson JJ, Sramkoski RM, Jacobberger JW, Zimmerman PA: Monitoring Plasmodium falciparu growth and development by UV flow cytometry using an optimized Hoechst-thiazole orange staining strategy. Cytometry A 2008, 73:546-554. 
25. Schmittgen TD, Livak K: Analyzing real-time PCR data by the comparative C(T) method. Nat Protoc 2008, 3:1101-1108.

26. Livak KJ, Schmittgen TD: Analysis of relative gene expression data using real-time quantitative PCR and the 2(-Delta Delta C(T)) Method. Methods 2001, 25:402-408.

27. Yim JJ, Ding L, Schaffer AA, Park GY, Shim YS, Holland SM: A microsatellite polymorphism in intron 2 of human Toll-like receptor 2 gene: functional implications and racial differences. FEMS Immunol Med Microbiol 2004, 40:163-169.

28. Johnson CM, Tapping Rl: Microbial products stimulate human Toll-like receptor 2 expression through histone modification surrounding a proximal NF-kappaB-binding site. J Biol Chem 2007, 282:31197-31205.

29. Severe falciparum malaria. World Health Organization, Communicable Diseases Cluster. Trans R Soc Trop Med Hyg 2000, 94(Suppl 1):S1-90.

30. Khor CC, Chapman SJ, Vannberg FO, Dunne A, Murphy C, Ling EY, Frodsham AJ, Walley AJ, Kyrieleis O, Khan A, Aucan C, Segal S, Moore CE, Knox K, Campbell SJ, Lienhardt C, Scott A, Aaby P, Sow OY, Grignani RT, Sillah J, Sirugo G, Peshu N, Williams TN, Maitland K, Davies RJ, Kwiatkowski DP, Day NP, Yala D, Crook DW, Marsh K, Berkley JA, O'Neill LA, Hill AV: A Mal functional variant is associated with protection against invasive pneumococcal disease, bacteremia, malaria and tuberculosis. Nat Genet 2007, 39:523-528.

doi:10.1186/1475-2875-11-47

Cite this article as: Greene et al:: Toll-like receptor polymorphisms and cerebral malaria: TLR2 $\triangle 22$ polymorphism is associated with protection from cerebral malaria in a case control study. Malaria Journal 2012 11:47.

\section{Submit your next manuscript to BioMed Central and take full advantage of:}

- Convenient online submission

- Thorough peer review

- No space constraints or color figure charges

- Immediate publication on acceptance

- Inclusion in PubMed, CAS, Scopus and Google Scholar

- Research which is freely available for redistribution

Submit your manuscript at www.biomedcentral.com/submit 